\title{
The transport properties of activated carbon fibers
}

\author{
S. L. di Vittorio \\ Department of Materials Science and Engineering, Massachusetts Institute of Technology, \\ Cambridge, Massachusetts 02139 \\ M. S. Dresselhaus \\ Department of Electrical Engineering and Computer Science and Department of Physics, \\ Massachusetts Institute of Technology, Cambridge, Massachusetts 02139 \\ M. Endo \\ Department of Electrical Engineering, Faculty of Engineering, Shinshu University, \\ Nagano 380, Japan \\ J-P. Issi, L. Piraux, and V. Bayot \\ Unité de Physico-Chimie et de Physique des Matériaux, Université Catholique de Louvain, \\ Louvain-la-Neuve, Belgium
}

(Received 18 October 1990; accepted 11 December 1990)

The transport properties of isotropic pitch-based carbon fibers with surface area $1000 \mathrm{~m}^{2} / \mathrm{g}$ have been investigated. We report preliminary results on the electrical conductivity, the magnetoresistance, the thermal conductivity, and the thermopower of these fibers as a function of temperature. Comparisons are made to transport properties of other disordered carbons.

\section{INTRODUCTION}

Activated carbons are characterized by their extremely high porosity and their large specific surface area, which can reach several thousands $\mathrm{m}^{2} / \mathrm{g}$. As a result, activated carbons are useful in applications where a large surface is required, such as filtration for anti-air pollution systems, the cleaning of drinking water, and blood filtering. Another potentially far-reaching application is to use activated carbons as the electrode material of new supercapacitors. ${ }^{1-3}$

Many techniques have been used to characterize activated carbons, such as electron microscopy, ${ }^{4} \mathrm{x}$-ray scattering, gas adsorption, ${ }^{5}$ and mercury porosimetry. ${ }^{5}$ From these analyses, the pore size distribution of the material is derived, and the pores are classified into micropores with diameters smaller than $2 \mathrm{~nm}$, mesopores with diameters between $2 \mathrm{~nm}$ and $50 \mathrm{~nm}$, and macropores larger than $50 \mathrm{~nm}$. In this paper, we characterize activated carbons using transport properties. We take advantage of the recent fabrication of activated carbon in fibrous form. Previously, activated carbon had been fabricated only in the form of grains or particles, which made quantitative transport measurements of this material impossible.

Transport properties can be used as a useful tool to characterize the activated carbon fibers (ACFs). Indeed, transport properties provide a measure of the relaxation time of the carriers (whether electrical charge carriers or heat carriers), yielding information on the density as well as the nature of defects. We expect two kinds of defects in ACFs: boundary scattering and scat- tering caused by the bulk defects related to the nongraphitic nature of the carbon material. Nonactivated carbon fibers have typically a specific surface area of $10 \mathrm{~m}^{2} / \mathrm{g}$, so that boundary scattering is negligible in these fibers. Boundary scattering, however, plays a significant role in ACFs, because of their huge specific surface areas. Whereas electron scattering is affected by local defects, phonons are scattered mainly by extended defects, such as dislocations, grain boundaries, and other boundaries, such as pores and surfaces. The use of the various transport experiments should therefore prove useful in analyzing the origin of scattering in the activated carbon fibers.

In this paper, we report measurements of the transport properties of a recently developed activated fiber derived from isotropic pitch, a precursor material that makes it possible to achieve a higher specific surface area (SSA) than with previously used precursor materials. The fiber studied in this paper has a SSA of $1000 \mathrm{~m}^{2} / \mathrm{g}$, which is a very high figure for any material. Indeed, in a very simple model where active carbon is described as a piling of a few graphene sheets with thickness $t$, the SSA turns out to be $2 / t \rho$, where $\rho$ is the density, which in single crystal graphite is $2.25 \mathrm{~g} / \mathrm{cm}^{3}$. On this basis, a SSA of $1000 \mathrm{~m}^{2} / \mathrm{g}$ yields a platelet thickness $t=9 \AA$. In other words, the porosity of the fiber studied in this paper corresponds roughly to a carbon material made out of sheets of carbon 3 atoms in thickness, with the sheets separated by voids. Using $3.35 \AA$ as the interplanar c-axis separation, a maximum SSA of 
$3000 \mathrm{~m}^{2} / \mathrm{g}$ is found for this simple model, which is comparable to the highest experimentally attainable SSA value.

The paper is divided into four sections focusing on each of the four transport measurements carried out on the ACFs: electrical conductivity, magnetoresistance, thermal conductivity, and thermopower. In each section, the data are analyzed and are compared to wellstudied nongraphitic carbons. When similar data were available, we have compared our results to as-grown ex-pitch fibers, in order to point out the novel features related to the high porosity. When such results could not be found, comparisons are drawn with other disordered carbons, such as glassy carbon, ${ }^{6}$ as-grown exPAN fibers, or as-grown vapor-deposited fibers. ${ }^{7}$ It should, however, be mentioned here that as-grown vapor-deposited fibers have a higher structural ordering than as-grown ex-pitch fibers or ex-PAN fibers.

\section{FABRICATION OF ACTIVATED CARBON FIBERS}

The fabrication of ACFs is a three-step process. Firstly, the fiber is formed by spinning the precursor material. Many precursor materials have been used to produce ACFs, such as phenolic materials, resins, polyacetate, cellulose, polyacrylonitrile (PAN), or pitch. In this paper, the precursor material was isotropic pitch. Prior to the activation process, the fiber undergoes an anti-flammable process at a temperature of 200 to $400{ }^{\circ} \mathrm{C}$. If a structure more complicated than a fiber, such as a felt, is desired, the material can be shaped before the anti-flammable process, or alternately right afterward. Next comes the activation process, which consists of heating the material in the temperature range $800-1200^{\circ} \mathrm{C}$ in the presence of $\mathrm{CO}_{2}$ or water vapor. This process removes some carbon through an oxidation reaction, removing also many impurities, and this process creates the highly porous structure. The main parameter that characterizes the activated carbon fibers is the specific surface area, expressed in $\mathrm{m}^{2} / \mathrm{g}$. It is measured using the absorption isotherms of $\mathrm{N}_{2}$ at $78 \mathrm{~K}$ and $\mathrm{CO}_{2}$ at $195 \mathrm{~K}$.

\section{EXPERIMENTAL DETAILS}

The samples used in these experiments consist of fibers about $1 \mathrm{~cm}$ long and $20 \mu \mathrm{m}$ in diameter, as determined by optical microscope measurements. In order to increase the thermal conductance of the sample, we carried out the thermal conductivity and thermopower experiments on a sample consisting of 40 fibers.

The electrical resistance of the fibers was measured under vacuum using a conventional DC four-contact technique. The typical contact resistance measured was $10 \mathrm{ohms}$. The measurements were carried out down to only $30 \mathrm{~K}$ because of the rapidly increasing resistance at low temperature. Going to lower temperatures would have required the use of an electrometer. The magnetoresistance experiments were carried out in the field range $0<H<5$ tesla on a single fiber using a superconducting magnet, and some of the results were also obtained with a conventional electromagnet.

The thermal conductivity and the thermopower of the sample were measured using the thermal potentiometer technique previously described by Piraux et al. ${ }^{8}$ In this technique, a four contact measurement is performed so as to eliminate the thermal contact resistances, very much in the same way as for electrical transport measurements. All leads connected to the sample were grounded on a heat sink, whose temperature was regulated so as to be the same as that of the sample, in order to minimize the heat leaks by conduction. In addition, the oven used to establish a thermal gradient along the sample was also shielded so as to reduce the losses by radiation.

The thermal properties were measured in the temperature range $55 \mathrm{~K}<T<300 \mathrm{~K}$. The thermal properties were not measured in the temperature range $4.2 \mathrm{~K}<T<55 \mathrm{~K}$ for two reasons. At the very lowest temperatures, where the heat capacity varies at $T^{3}$, the thermal conductance was too small to be measurable. At intermediate temperatures, the time needed to reach thermal equilibrium (which is inversely proportional to the conductance) was too long for practical measurements.

\section{EXPERIMENTAL RESULTS}

\section{A. Electrical conductivity}

Results for the temperature dependence of the electrical conductivity are shown by the points in Fig. 1(a). The magnitude of the room temperature resistivity $\left(2 \times 10^{-3} \Omega \cdot \mathrm{m}\right)$ is higher than that of any previously reported carbon fiber. For comparison, we estimated from Ref. 7 the resistivity $\left(7 \times 10^{-5} \Omega \cdot \mathrm{m}\right)$ of an ex-pitch fiber heat-treated to $1000{ }^{\circ} \mathrm{C}$, which is the range of temperature used for the activation process $\left(800^{\circ} \mathrm{C}<T_{H T}<1200{ }^{\circ} \mathrm{C}\right)$. Although we used for this estimate a result given for ex-mesophase pitch fibers, since no data were available for isotropic pitch, the large difference in resistivity indicates that the high porosity plays a major role in determining the electrical properties of the ACFs. In such a highly resistive material, a very important question is whether the material is homogeneous, and whether the current is carried by the bulk of the fiber, instead of, say, a thin surface layer. Based on TEM pictures, ${ }^{9}$ the structure of the surface of the fiber looks very similar to that of the bulk. Thus, we are confident that we are indeed measuring the bulk resistivity of the fiber. As the temperature is lowered, the resistivity increases steeply, which is very 


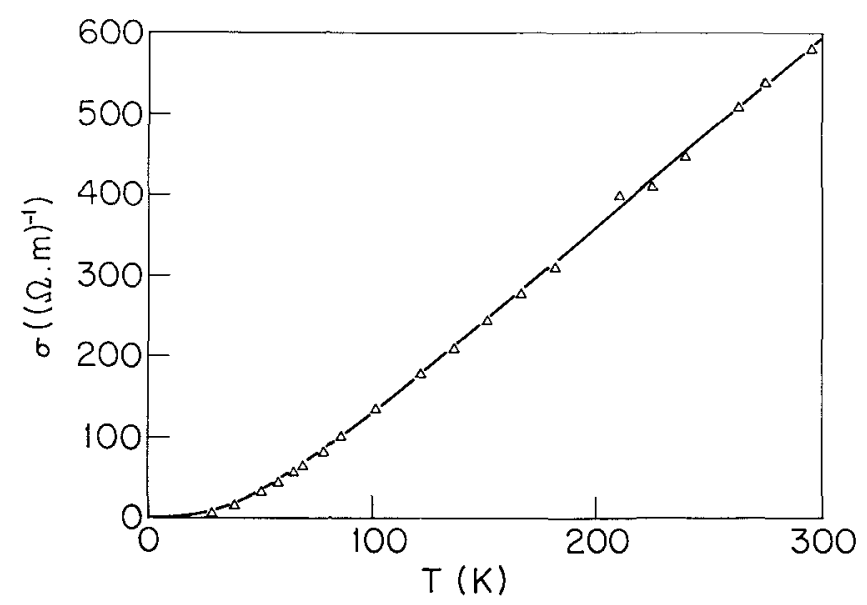

(a)

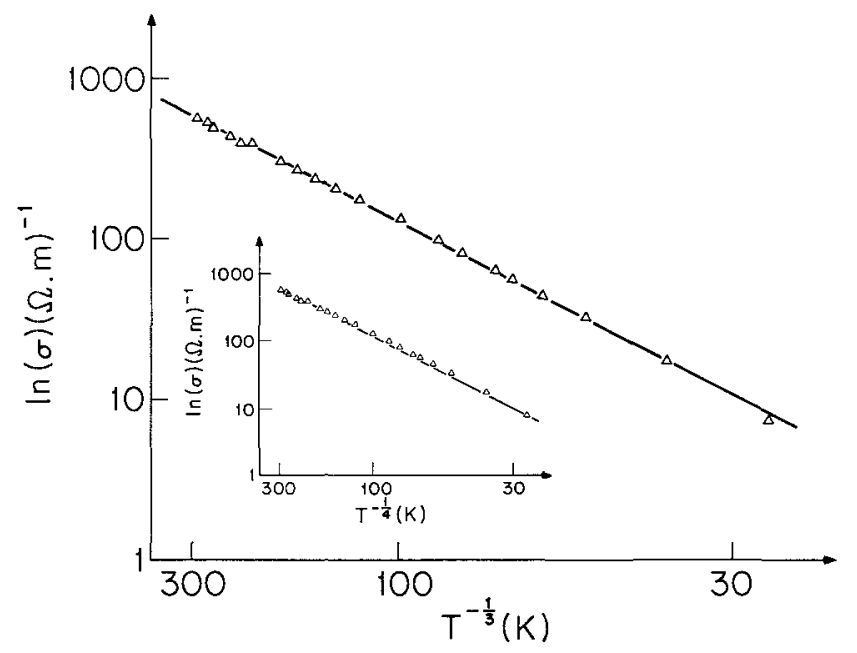

(b)

FIG. 1. (a) The temperature dependence of the conductivity from $20 \mathrm{~K}$ to room temperature for a pitch-based activated carbon fiber (specific surface area $1000 \mathrm{~m}^{2} / \mathrm{g}$ ). The dotted line shows the fit to a 2D variable-range hopping model. (b) Plot of $\ln \sigma(T)$ vs $T^{-1 / 3}$ for the same fiber as in (a). The inset to (b) shows a plot of the same fiber for $\ln \sigma(T)$ vs $T^{-1 / 4}$.

similar to the behavior in glassy carbon. This general behavior is also observed in ex-PAN and ex-pitch fibers with heat treatment temperature $T_{H T}<1000{ }^{\circ} \mathrm{C}$. In contrast, the benzene-derived fibers have a nearly flat resistivity in the low to room temperature range, characteristic of a better structural ordering in these fibers.

Depending on the temperature and the amount of disorder in the material, we expect various laws to govern the temperature dependence of the resistivity. The precursor pitch material had been heat-treated to temperatures of $800{ }^{\circ} \mathrm{C}<T_{H T}<1200{ }^{\circ} \mathrm{C}$, so that the original organic molecules had been decomposed and our material is essentially made out of carbon. As demonstrated by the predominantly positive room temperature thermopower $S$ (to be discussed below), the high density of defects (or surface states) results in a predominantly $p$-type material, with a large density of holes. The chemisorption of other chemical species, mainly oxygen, on the huge surface of the activated carbon fiber is another possible mechanism for charge transfer and the creation of positively charged localized states. As opposed to single crystal graphite or turbostratic graphite, the ACFs do not have the simple band structure of a semimetal or a nearly-compensated semiconductor. The appropriate electronic model for describing activated carbon is a density of states populated by a large density of holes, with the states at the Fermi level being localized because of the high porosityinduced disorder.

Within this general model, several more specific competing mechanisms can account for the observed temperature-dependent resistivity. At high temperature, thermal excitation of electrons from the Fermi level to the mobility edge will populate some extended states, although the disorder is so high in ACFs that it is possible that all states are localized, and that a mobility edge does not exist. At lower temperature, thermal excitation is unlikely and transport occurs through a carrier variable-range hopping mechanism. ${ }^{10}$ When both mechanisms are taken into account, the following general law for the electrical conductivity is obtained:

$$
\sigma=\sigma_{0} \exp \left[-\left(\frac{T}{T_{0}}\right)^{-n}\right]+\sigma_{1} \exp \left[-\left(\frac{\epsilon_{1}}{\mathrm{k} T}\right)\right]
$$

where $\sigma_{0}$ and $\sigma_{1}$ are the conductivities at infinite temperature for the two processes considered, $T_{0}$ is a temperature related to the localization length, and $\epsilon_{1}$ is the energy difference between the mobility edge and the Fermi level, assuming that a mobility edge can be identified. The first term in Eq. (1) is thus the variablerange hopping term, whereas the last term describes thermal excitation to the mobility edge. The variable $n$ in Eq. (1) depends on the dimensionality of the transport, unless carrier-carrier correlation effects are dominant, in which case the exponent $n$ is modified to $1 / 2$ independent of the dimensionality of the transport processes.

Because of the flexibility of Eq. (1), it can be well fit to the experimental $\sigma(T)$ data for ACFs and other disordered carbons. Some workers ${ }^{11}$ have, for example, used more than one thermal excitation term to describe more than one thermally-activated process. Baker and Bragg $^{12}$ also added a weak localization term to Eq. (1) to fit the experimental conductivity data of amorphous carbons. However, this new analytical term was closely related to the observed microstructure, and is thus irrelevant for ACFs. Thus, Eq. (1) provides a broad framework for the interpretation of $\sigma(T)$ data for disordered carbons. On the other hand, with this flexibility comes nonuniqueness, and the resulting fitting parameters depend on the somewhat arbitrary choice of a fitting model. 
In this paper, the experimental temperaturedependent conductivity has been fitted to the first term of Eq. (1), to reduce the number of adjustable parameters and because the second term was not needed to get a good fit. This small second term corresponds to a system that is deeply insulating, i.e., where there is a large energy difference between the Fermi energy and the mobility edge, or where a mobility edge may not even exist. This deeply insulating behavior is consistent with the very high disorder observed in ACFs. The microstructure of activated carbons as observed using TEM by Huttepain and Oberlin ${ }^{4}$ indicates a structure formed of crumpled carbon sheets. Activated carbon is described as made up of continuous stacks of aromatic layers very distorted and entangled, and with very high radii of curvature. This is an indication that transport in activated carbons is two-dimensional, at least partially, suggesting use of the exponent $n=1 / 3$ in Eq. (1). This $1 / 3$ exponent is also found to provide the best fit to the experimental data among the three $1 / 2,1 / 3$, and $1 / 4$ exponents, corresponding, respectively, to $1 \mathrm{D}, 2 \mathrm{D}$, and $3 \mathrm{D}$ variable range hopping. The results obtained for the curve-fitting to the exponents $n=1 / 3$ and $n=1 / 4$ are shown in Fig. 1(b). In Fig. 1(a), the experimental results are shown as triangles, and the solid lines are the theoretical fits to the data points. From the fit, we extract values $\sigma_{0}=1.97 \times 10^{4}(\Omega \cdot \mathrm{m})^{-1}$ and $T_{0}=$ $1.27 \times 10^{4} \mathrm{~K}$. Following Mott, ${ }^{10}$ we obtain an estimate for the localization length in activated carbon fibers using the formula:

$$
\xi=\sqrt{\frac{27}{\pi N\left(E_{\mathrm{F}}\right) \mathrm{k} T_{0}}}
$$

where $N\left(E_{\mathrm{F}}\right)$ is the density of states at the Fermi level. To evaluate $\xi$, we use the value of $T_{0}$ extracted from the conductivity data, and the density of states for single crystal graphite away from the band edge, which is approximately linear in energy. Following Ref. 7 , we use the formula for the $2 \mathrm{D}$ density of states:

$$
N(E)=\frac{8|E|}{3 \pi a_{0}^{2} \gamma_{0}^{2}}
$$

where $a_{0}=2.462 \AA$ is the in-plane lattice constant and $\gamma_{0}=3.16 \pm 0.05 \mathrm{eV}$ is the nearest neighbor overlap energy in single crystal graphite. ${ }^{7}$ As indicated by the small value of the thermopower (discussed below), the carrier density is large, owing to the large concentration of defects in the ACFs. To obtain an estimate for $\xi$, we chose a carrier concentration of $10^{-2}$ carrier per carbon atom, as compared to $10^{-4}$ carrier per atom in graphite. This value is quite arbitrary, but it is not critical since the localization length depends only weakly (as the fourth root, actually) on the carrier density. We thus find an estimate for the localization length $\xi \sim 20 \AA$, which is on the same order as the average distance between pores. ${ }^{9}$ The value found for $\xi$ is an indication that the localization phenomenon is induced by the random potential created by the presence of pores in the material, or by the presence of dangling bonds in the pores. The small value of the localization length also indicates that coulombic correlation $[n=1 / 2$ in Eq. (1)] might play an important role in the conduction at very low temperatures.

More conclusive results could be obtained by correlating the localization length with the average distance between pores for several ACFs with different specific surface areas. In the particular fiber studied in this paper, the average distance between pores is approximately equal to the average pore radius, as measured by TEM micrographs. ${ }^{9}$ With increasing specific surface area, however, the average pore dimension remains roughly constant, whereas the density of pores increases. ${ }^{9}$ Thus the average distance between pores is expected to decrease, and we therefore expect the localization length to decrease with increasing specific surface area. Though much information can be obtained from $\sigma(T)$ studies on ACFs, systematic studies are needed to address the issue of uniqueness of the fit to Eq. (1), and experiments using other techniques are also needed to delineate the transport mechanisms more reliably.

\section{B. Magnetoresistance}

We also performed transverse magnetoresistance measurements in the field range $0<H<5$ tesla at various temperatures, as shown in Fig. 2, where solid lines are used to connect the experimental points. The

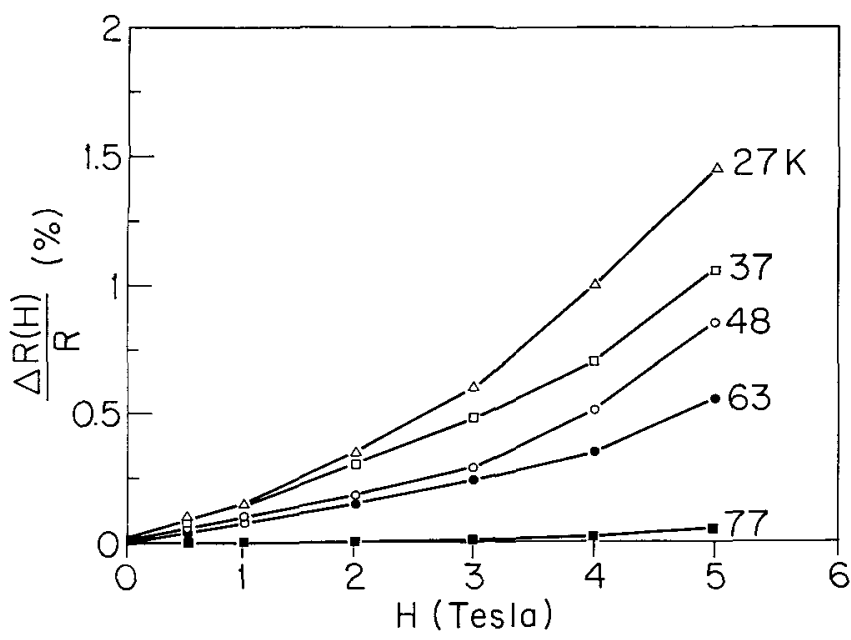

FIG. 2. The transverse magnetoresistance of activated carbon fibers from 0 to 5 tesla for several temperatures in the range $27 \mathrm{~K}$ to liquid nitrogen temperature. Note the positive sign of the magnetoresistance over the whole field range measured. 
magnetoresistance is weak and positive, increasing in magnitude with decreasing temperature. This behavior of the magnetoresistance is a general feature of highly disordered carbons, such as ex-PAN and ex-pitch fibers, but also of glassy carbon. ${ }^{7,13}$ As readily seen from the temperature dependence of the conductivity, the transport properties of ACF cannot be understood within the framework of band theory, and the positive magnetoresistance in Fig. 2 does not correspond to the conventional quadratic increase in field associated with band theory $\rho(H)=\rho(0) \times\left[1+(\mu H)^{n}\right]$ with $n=2$. As of now, the origin of the positive magnetoresistance observed in Fig. 2 is not well understood. A power law fit of the magnetoresistance curves yields exponents consistently in the $n=1.3$ to $n=1.8$ range. For very disordered ex-PAN fibers, Hambourger ${ }^{14}$ showed that the magnetoresistance was positive and nearly isotropic. In light of Hambourger's result, a comparison of the longitudinal and transverse magnetoresistance would be instructive. Indeed, a recent theory of the magnetoresistance in the variable-range hopping regime ${ }^{15}$ predicts a quadratic increase in the resistivity at low fields, and a saturation at higher fields for materials in the variablerange hopping regime but far away from the metalinsulator transition. This theory, however, predicts a vanishing longitudinal magnetoresistance, as opposed to the result of Ref. 14. Thus detailed studies of the magnetoresistance of ACFs could be of interest.

\section{Thermal conductivity}

The temperature dependence of the thermal conductance $\kappa(T)$ of the ACF fibers has been measured for $55 \mathrm{~K}<T<300 \mathrm{~K}$. The results closely follow a $T^{3}$ analytical dependence in the temperature range $85 \mathrm{~K}<$ $T<300 \mathrm{~K}$. This $T^{3}$ law is characteristic of thermal conduction by radiation during the experiment. It is not an intrinsic property of the material, but is found whenever the thermal conductance measurement of the sample is dominated by the radiative exchanges between the parts of the measuring apparatus, which are not all at the same temperature. For temperatures in the $55 \mathrm{~K}<T<85 \mathrm{~K}$ range, the $\kappa(T)$ curve deviates substantially from a $T^{3}$ law, showing that in this temperature range, the measurements are no longer dominated by radiation effects. This allows us to estimate an upper limit for $\kappa(T)$ for $55 \mathrm{~K}<T<85 \mathrm{~K}$. To make this estimate, we have fitted the measured $\kappa(T)$ curve between $100 \mathrm{~K}$ and $300 \mathrm{~K}$ to a $T^{3}$ law and we have then subtracted the $\kappa(T)$ curve thus determined from the measurements in the $55 \mathrm{~K}<T<85 \mathrm{~K}$ range. We therefore estimate an upper limit for the thermal conductivity of the fibers around liquid nitrogen temperature of $0.25 \mathrm{~W} / \mathrm{m} \cdot \mathrm{K}$, which is an order of magnitude lower than for as-grown ex-PAN fibers. ${ }^{16}$ This low value for $\kappa$ at $77 \mathrm{~K}$ also proves that boundary scattering plays a major role in the scattering of phonons as well as in electron scattering in ACFs, as expected. Using the Wiedemann-Franz relationship, we find an estimate for the electronic contribution to the thermal conductivity of $10^{-4} \mathrm{~W} / \mathrm{m} \cdot \mathrm{K}$. Thus we conclude that the thermal conductivity in ACFs is very low and that the heat in these materials is carried by phonons, whereas the electrons hardly play any role in heat transport.

\section{Thermopower}

Results for the temperature dependence of the thermopower $S$ between $55 \mathrm{~K}$ and room temperature are displayed in Fig. 3. Because of the low thermal conductance of the fibers, the thermopower is very difficult to measure, and we estimate an error-bar of $\pm 0.2 \mu \mathrm{V} / \mathrm{K}$, substantially higher than the estimated error for better conducting materials. The thermopower of ACF, as shown in Fig. 3, is weakly positive above $150 \mathrm{~K}$ and weakly negative below this temperature. At much lower temperatures, we expect the magnitude of the thermopower to decrease, since $S=0$ at $T=0 \mathrm{~K}$. The general behavior for the experimental points for $S(T)$ in Fig. 3 is very similar to that found by Endo et al. ${ }^{17}$ for as-grown benzene-derived carbon fibers (dashed curve). $S(T)$ for the as-grown benzene-derived carbon fibers shows a positive thermopower for temperatures above $140 \mathrm{~K}$ and weakly negative below, with an approximately linear region from $50 \mathrm{~K}$ to room temperature. These thermopower results prove that conduction in ACFs at room temperature is hole-dominated, similar to other disordered carbon materials, ${ }^{16}$ whereas mixed carrier conduction takes place at low temperatures. The small magnitude of $S$ indicates that the carrier density is large. However, from the $\sigma(T)$ measurements, we know that the carriers are localized. In

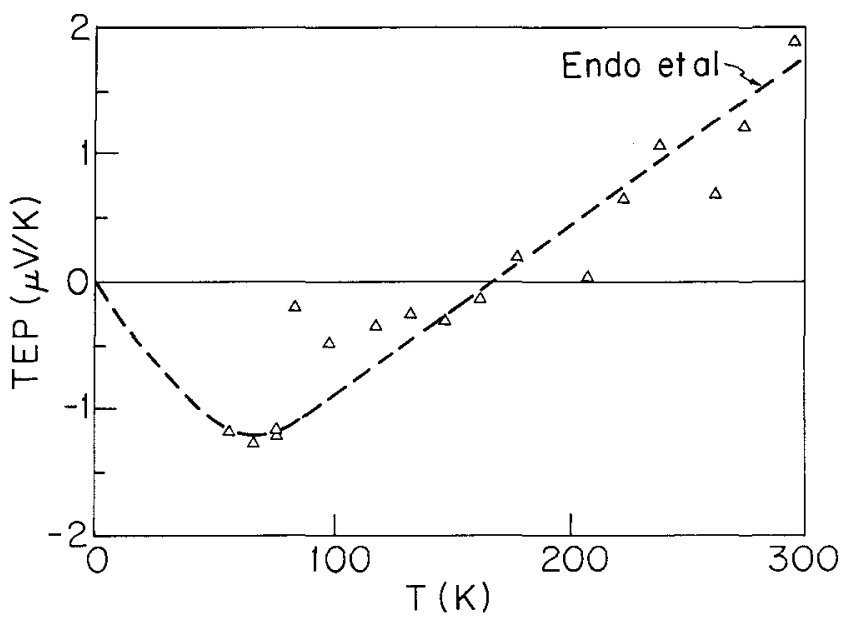

FIG. 3. The temperature dependence of the thermopower in the temperature range $55 \mathrm{~K}$ to room temperature. The dashed line represents the result of Endo et al. in Ref. 17 for as-grown vapordeposited carbon fibers. 
physical terms, a large density of dangling bonds is associated with the pores of the ACFs. The weak negative thermopower at low temperatures is similar to the results found by Endo et al. ${ }^{17}$ in as-grown vapor-deposited fibers, and was attributed in that work to the donor behavior of the iron spheres used to catalyze the fiber growth from the vapor phase. It is not clear as of now if this negative thermopower at low temperatures is a more general feature of disordered carbons than was indicated in this early work. ${ }^{17}$

\section{CONCLUDING COMMENTS}

Preliminary measurements of the electrical and thermal transport properties of activated carbon fibers with surface area $1000 \mathrm{~m}^{2} / \mathrm{g}$ have been reported. As expected from the large surface area, boundary scattering is very strong and the activated fibers are found to be highly resistive, both electrically and thermally. We have shown that the defects associated with the porosity play a very important role in determining the thermal and electrical transport properties. Quantitative studies are needed to separate the effect of disorder in the carbon material from the effect of the large surface area.

As stated above, all measurements in this paper were performed in vacuum. Also of interest would be the study of the electrical properties of activated carbon fibers as a function of the amount of gas adsorbed or trapped in the pores and also of the chemical species of the adsorbates. The ACFs clearly have a high density of dangling bonds, and the transport properties are likely to be sensitive to the way in which these bonds are terminated. Since thermal measurements have to be carried out in vacuum, thermal transport studies on fibers with adsorbates would be very difficult. With regard to the electrical transport, we have observed a change on the order of a few percent in the electrical resistivity of the fibers in air relative to vacuum.

\section{ACKNOWLEDGMENTS}

We would like to thank Dr. G. Dresselhaus for many stimulating discussions. The MIT authors grate- fully acknowledge subcontract \#B-130530 from the Lawrence Livermore National Laboratory for support of this research. L.P. acknowledges financial support from the Fonds National Belge de la Recherche Scientifique. The work performed in Louvain-la-Neuve was within the framework of the "Action de Recherche Concerte" program sponsored by the Belgian Ministry of Scientific Policy.

\section{REFERENCES}

${ }^{1}$ M. Endo, Y. Okada, and H. Nakamura, Synth. Metals 34, 739 (1989).

${ }^{2}$ I. Tanahashi, A. Yoshida, and A. Nishina, Carbon 28, 477 (1990).

${ }^{3}$ A. Yoshida, I. Tanahashi, Y. Takeuchi, and A. Nishino, IEEE

Transactions on components, hybrids and manufacturing technology, CHMT-10 1, 100 (1987).

${ }^{4} \mathrm{M}$. Huttepain and A. Oberlin, Carbon 28, 103 (1990).

${ }^{5}$ R. P. Bansal, J-B. Donnet, and F. Stoeckli, Active Carbon (Marcel Dekker, New York, 1988).

${ }^{6}$ G. M. Jenkins and K. Kawamura, Nature 231, 175 (1971).

${ }^{7}$ M. S. Dresselhaus, G. Dresselhaus, K. Sugihara, I. L. Spain, and H. A. Goldberg, Graphite Fibers and Filaments (Springer-Verlag, 1988).

${ }^{8}$ L. Piraux, J-P. Issi, and P. Coopmans, Measurement 5, 2 (1987).

${ }^{9} \mathrm{M}$. Endo (private communication).

${ }^{10} \mathrm{~N}$. Mott, Conduction in non-crystalline materials (Clarendon Press, Oxford, 1987).

${ }^{11} \mathrm{M}$. Endo, A. Kato, H. Ueno, and M. Shiraishi, Transaction of the Institute of Electrical Engineers of Japan 108-A, No. 7, 279 (1988).

${ }^{12}$ D. F. Baker and R. H. Bragg, Phys. Rev. B 28, 2219 (1983).

${ }^{13}$ D. Robson, F.Y. I. Assabghy, E. G. Cooper, and D. J. E. Ingram, J. Phys. D6, 1822 (1973).

${ }^{14}$ P. D. Hambourger, Appl. Phys. Comm. 5, 223 (1986).

${ }^{15}$ U. Sivan, O. Entin-Wohlmann, and Y. Imry, Phys. Rev. Lett. 60, 1566 (1988).

${ }^{16}$ J. Heremans and C. P. Beetz, Jr., Phys. Rev. B 32, 1981 (1985).

${ }^{17}$ M. Endo, I. Tamagawa, and T. Koyama, Jpn. J. Appl. Phys. 16, $1771(1977)$. 